\title{
Lack of evidence for a tactual Poggendorff illusion
}

\author{
PETER WENDEROTH and DAVID ALAIS \\ University of Sydney, Sydney, New South Wales, Australia
}

\begin{abstract}
Lucca, Dellantonio, and Riggio (1986) reported large distortions in a tactual analogue of the visual Poggendorff illusion. They also reported large effects in the direction opposite to the visual illusion, which they termed "inversions." However, their evidence for such effects is questionable; they used what we consider to be inappropriate measurement and analysis procedures. In attempting to replicate their experiment, and in conducting four additional experiments, we found no evidence at all for their alleged tactual analogue of the visual Poggendorff effect. Instead, we demonstrated that "inversions" are likely due to the use of a raised stimulus display that causes artifactual mistracking, which is totally unrelated to normal mechanisms of alignment judgment. We also discuss the possible role of intersensory factors in the generation of tactual illusions.
\end{abstract}

For many years, researchers have attempted to study tactual analogues of visual illusions, often in blind and in blindfolded but sighted subjects (e.g., Bean, 1938; Hatwell, 1960; Patterson \& Deffenbacher, 1972; Rèvèsz, 1934; Tsai, 1967). Part of the rationale for such experiments is that a high correlation between tactual and visual illusions would cast doubt on any theory couched purely in terms of visual mechanisms (Over, 1968). It nevertheless would remain possible that similar processes operate in the two domains (e.g., lateral inhibition) or that performance in one modality was not based primarily on factors intrinsic to that modality. For example, Appelle and Gravetter (1985) presented convincing evidence that a haptic oblique effect (poorer acuity for oblique than for vertical or horizontal orientation) was similar to its visual counterpart, primarily because subjects use visual imagery in making haptic orientation judgments.

Recently, Lucca, Dellantonio, and Riggio (1986) had blindfolded subjects use two fingers actively to explore Poggendorff-type figures constructed from raised aluminum strips. Both right and left hands were used to explore four figures, such as those represented in Figure 1. Further details of the Lucca et al. apparatus and method need not be considered here, since our first experiment is an attempt to replicate theirs.

Lucca et al. (1986) claimed to have obtained large illusions (of the order of $4 \mathrm{~mm}$ ) in the full Poggendorff figure with parallels (P2 and P1 in Figure 1) but small effects (of the order of $1 \mathrm{~mm}$ ) in the parallel-less ("simple alignment") figures (AL1 and AL2 in Figure 1). More importantly, they claimed to have found that the majority

This research was supported by an Australian Research Council Grant A78831871 to P. Wenderoth. Correspondence should be addressed to Peter Wenderoth, Department of Psychology, University of Sydney, Sydney, NSW 2006, Australia. of these large effects with parallels were in the direction opposite to the usual visual effect ("inversions," in their terms). Thus, whereas the truly aligned obliques in, say, Figure $1(\mathrm{P} 2)$ appear visually misaligned such that the upper right oblique appears too high and the lower left oblique appears too low, Lucca et al. found that tactually the lower left oblique was set lower than physical alignment. That is, it appeared too high. If this were the case, it would be extremely important for perceptual theory, because it would suggest that quite different mechanisms mediate judgments of these simple figures in the visual and tactile domains.

Scrutiny of the Lucca et al. (1986) paper reveals that the data they present are not really informative regarding the magnitude or the direction of the tactual Poggendorff illusion. First, to obtain their overall mean illusions, they averaged the unsigned setting errors, as did Witkin and Asch (1948) many years ago in their experiments on the rod-and-frame illusion. But, as Wenderoth (1974) pointed out, the unsigned error is more a measure of the variance of settings than of their central tendency; the mean of a set of scores might be zero, yet the average unsigned error must be positive if the scores show any variability at all. So Lucca et al.'s (1986) measure of the average tactual Poggendorff effect tells us nothing about the magnitude and direction of illusions.

Second, Lucca et al. (1986) referred to previous work, some of which was their own, which had revealed "inversions," settings in the direction opposite the usual visual effects. Following a procedure adopted by Pasnak and Ahr (1970), Lucca et al. (1986) then calculated the mean of all the individual settings that were in the same direction as the visual effect (here called positive effects) and, separately, the mean of all the negative settings. They reported mean positive effects near zero but mean "inversions" of 3-4 mm. Again, these are measures more closely related to the variance of the scores; a subject with 


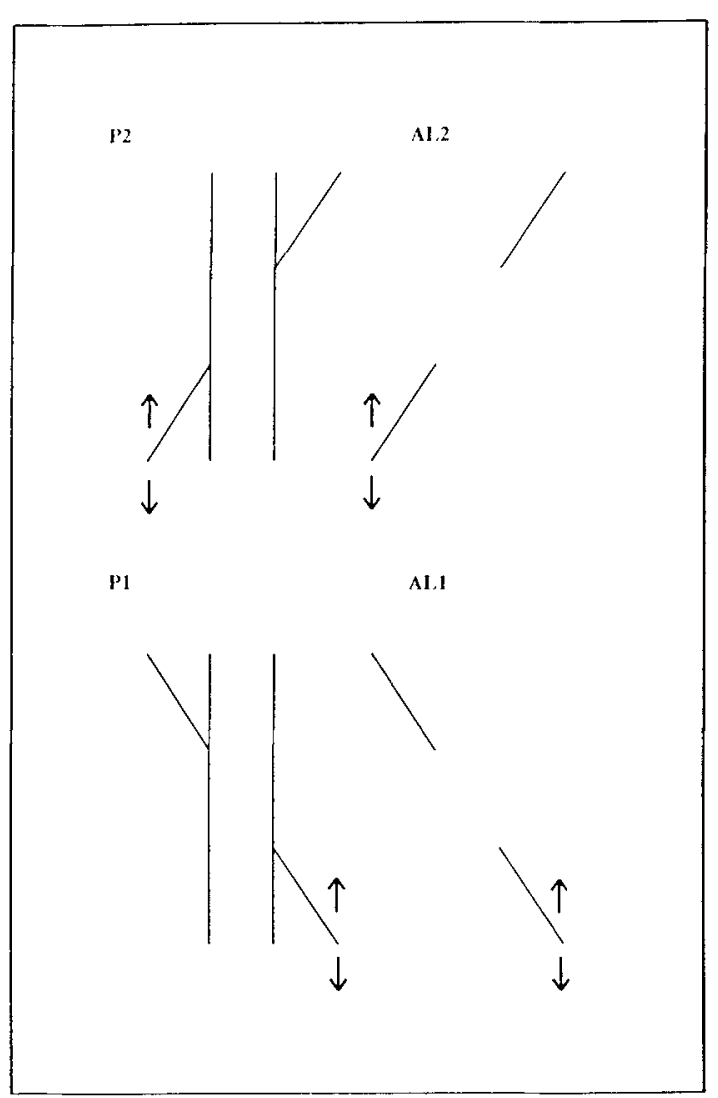

Figure 1. Schematic representation of stimuli used by Lucca, Dellantonio, and Riggio (1986). Alignment (AL) and Poggendorff (P) figures with transversals oriented $45^{\circ}$ (2) or $135^{\circ}$ (1). In each case, subjects move the lower segment (arrows) into apparent alignment with the opposite transversal.

five settings of $+2 \mathrm{~mm}$ and five of $-2 \mathrm{~mm}$ would have a mean error of zero. Yet Lucca et al. would have reported this as a positive effect of $2 \mathrm{~mm}$ and an "inversion" effect of $2 \mathrm{~mm}$.

It is common in studies of the Poggendorff effect to subtract mean errors obtained with the parallel-less figure (AL1 and 2 in Figure 1) from those obtained with the full figure (P1 and 2 in Figure 1), in order to obtain a measure that reflects the effect of the parallels. Lucca et al. (1986) did not do this, and the data they presented allowed no such analysis to be made. After contacting the authors in an unsuccessful attempt to obtain the raw data, which unfortunately were no longer available, we decided to replicate the experiment.

\section{EXPERIMENT 1}

\section{Method}

Apparatus. The dimensions of the stimulus figures used, duplicating those of Lucca et al. (1986), are shown in Figure 2, where the size of the total base $(330 \times 350 \mathrm{~mm})$ is not drawn to scale. There were four such pieces of apparatus, one for each display in Figure 1, although Figure 2 shows only the template for AL2 and P2. The wooden base was presented flat on a table to which it was firmly held. Two straight tracks were routed into the base, and these ran from top to bottom. All stimulus displays were divided into two halves, and each half had lubricated pins on the underside that fitted into the tracks on the base, allowing either half to slide smoothly up and down along the base. Only one half was free to move in any condition. The moving half moved smoothly along the vertical track, but not so easily that large overshooting might occur. The $350 \times 330 \mathrm{~mm}$ stimulus background was constructed of $6-\mathrm{mm}$-thick PVC plastic to make the background as featureless as possible. Slots in the shape of the illusion figures were cut in the background, and sheets of $1.5-\mathrm{mm}$ PVC plastic were pushed up through these slots until they protruded $1 \mathrm{~mm}$ vertically, where they were fixed in place. The wooden base could be moved to present a pattern to either the right or the left hand.

Each subject's forearm was held firm at the wrist and elbow by two Velcro straps $200 \mathrm{~mm}$ apart and mounted on a $350-\mathrm{mm}$ length of wood, $75 \mathrm{~mm}$ in width and $50 \mathrm{~mm}$ in height, allowing the hand a comfortable position with maximum freedom to explore each pattern. The forearm was aligned with the center of the stimulus pattern.

Following Lucca et al. (1986), we refer to the four stimuli as AL (alignment, no parallels) or $\mathrm{P}$ (Poggendorff, with parallels), and " 1 " and " 2 " refer to the transversal orientation, which was either $135^{\circ}(1)$ or $45^{\circ}(2)$. Considerable care was taken to ensure that all pattern segments were evenly raised by $1 \mathrm{~mm}$, that all protrusions were smooth and free of distracting features, and that transversals exactly abutted the parallels without any discernible gap.

In all cases, the subject moved the transversal closest to the body midline, and the fixed transversal and the parallels, where present, were contained entirely in the nonmovable section of the apparatus. The backboard was large, to ensure that subjects made no contact with the edges, and the experimenter monitored this behavior throughout.

Procedure. The subjects were first shown a sketch of a transversal pair, and the task was explained. The sketch was very rough, without parallels, so that there was little likelihood that any visual memory of the stimulus would affect the tactual task. This procedure was adopted because we assumed that Lucca et al. (1986) must have done something similar; otherwise, the verbatim instructions they quoted (p. 376) would have been difficult for the blindfolded subject to comprehend. After being blindfolded, the subjects sat facing the apparatus and were presented with an $A L$ and a $P$ pattern, one to each hand, which they traced completely to identify the patterns.

Five practice trials were given to each hand with an AL figure. The instruction was to trace from the variable to the fixed transversal a number of times until the two appeared to be aligned. After each trace, the subject's middle and index fingers only were placed back on the variabłe segment until the subject was satisfied that alignment had been achieved. It was stressed that the task was extremely difficult and that accuracy was essential, although settings were required to be completed within $15 \mathrm{sec}$. Because the fixed and variable halves of the background were flush when the transversals were aligned, a scale calibrated in $0.5-\mathrm{mm}$ steps on the PVC background allowed illusions to be read off, which were called positive if they were in the visual Poggendorff direction and negative otherwise.

Each subject completed eight conditions: four figures (Figure 1) $\times 2$ hands. There were 10 trials in each condition, with starting positions around collinearity of $\pm 0, \pm 2, \pm 4, \pm 6$, and $\pm 8 \mathrm{~mm}$, presented in random order. The left- and right-hand trials were intermingled so that, for any one figure, the 20 trials were completed in five consecutive sets of RLLR. The four different stimulus figures were presented in random order. These procedures were designed to follow those of Lucca et al. (1986) as closely as could be determined.

Subjects. Fifteen students from an introductory psychology course acted as subjects, in return for nominal course credit. All were righthanded. 


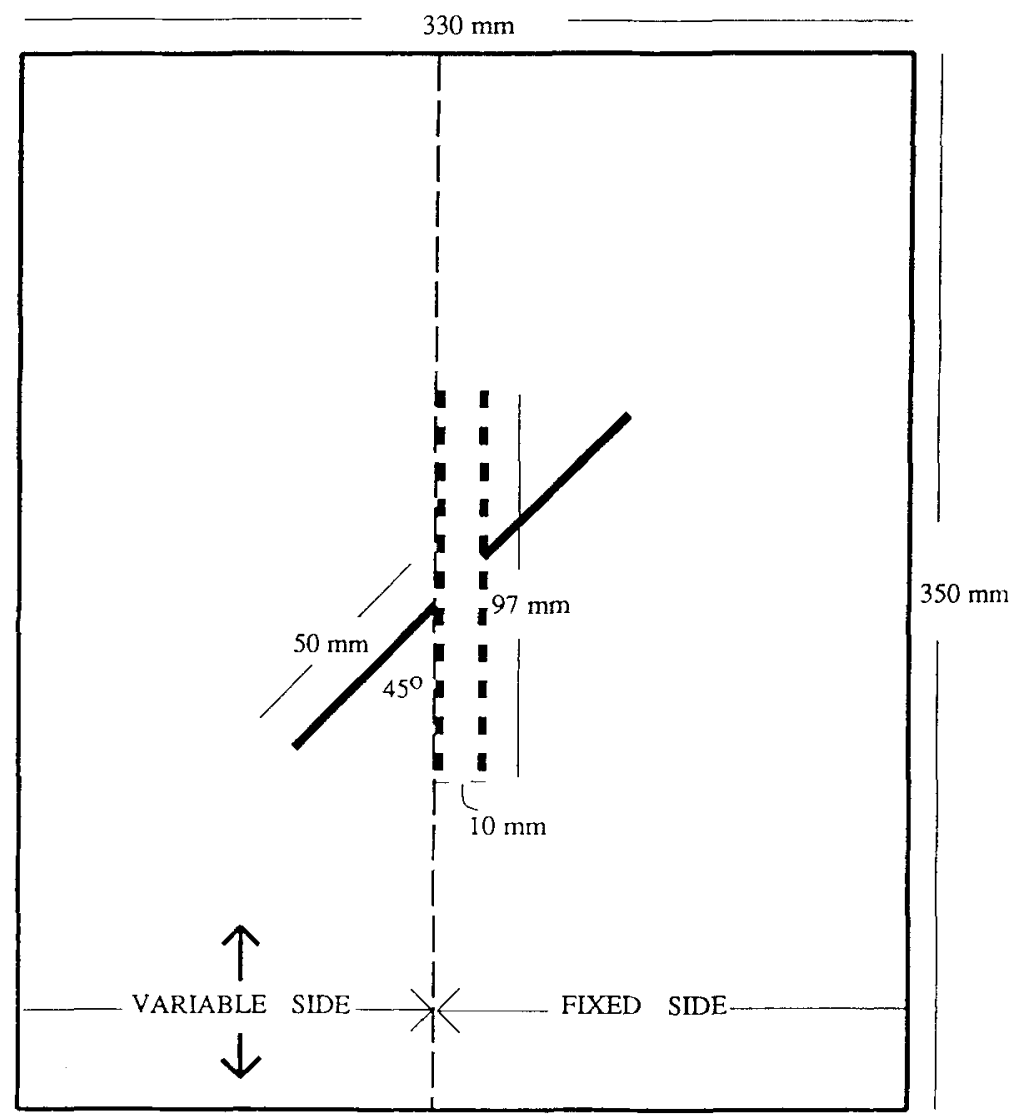

Figure 2. Schematic representation of construction of stimuli used in Experiment 1. Only the AL2 and P2 template is shown. Dimensions are to scale except for backboard edges.

\section{Results}

The means and standard errors of alignment settings are shown for each of the eight conditions in the body of Figure 3. None of these individual means was different from zero, even with a one-tailed $t$ test, with $p(14)>.05$ in every case. It follows that, since the $\mathrm{AL}$ and $\mathrm{P}$ means were in the same direction for either hand, the test minus pretest (P minus $\mathrm{AL}$ ) means would also not be significant.

Figures $4 \mathrm{~A}-4 \mathrm{~B}$ present a comparison of the absolute errors obtained by us and by Lucca et al. (1986), respectively. Naturally the errors are all positive, as discussed in the introduction, and comparison of Figures 3 and $4 \mathrm{~A}$ simply illustrates the fact that absolute errors bear little relation to the means.

It is noticeable that, for our data, the standard errors associated with the absolute errors are larger than those of Lucca et al. (1986). Part of the reason for this is that our sample size was smaller, but our standard deviations were also in excess of theirs. This issue is addressed in Experiments 2-5. The inset in Figure 3 shows the Lucca et al. "illusion" scores-that is, absolute errors of positive settings and absolute errors of negative settings. In contrast to the results of Lucca et al., which exhibited almost zero positive $P$ effects and 3-4-mm "inversion" effects, we obtained similar "inversion" data but much larger positive effects. Inspection of our raw data indicated that the range of positive effects was much larger than the range of "inversion" effects. For P1, "inversion" effects ranged from -0.8 to $-7.0 \mathrm{~mm}$, whereas positive effects ranged from 0.4 to $9.0 \mathrm{~mm}$. For $\mathrm{P} 2$, the corresponding ranges were -0.1 to $-6.2 \mathrm{~mm}$ and 0.3 to $-9.0 \mathrm{~mm}$. Possible reasons for the extreme positive effects are discussed below.

\section{EXPERIMENT 2}

In Experiment 1, most subjects approached the task in a stepwise procedure, adjusting a little at a time until satisfied. This meant that some subjects (confirmed by postexperiment debriefing) had not completed settings after the allowed $15 \mathrm{sec}$. Heller (1984) has reported that tactual form recognition of cookie-cutter shapes jumped from $70.7 \%$ to $95.2 \%$ as exposure time increased from 5 to $30 \mathrm{sec}$. Also, our subjects reported that it seemed unnatural to make judgments by proceeding from the adjustable to the fixed transversal and that the reverse would have been more natural. In Experiment 2, therefore, we 


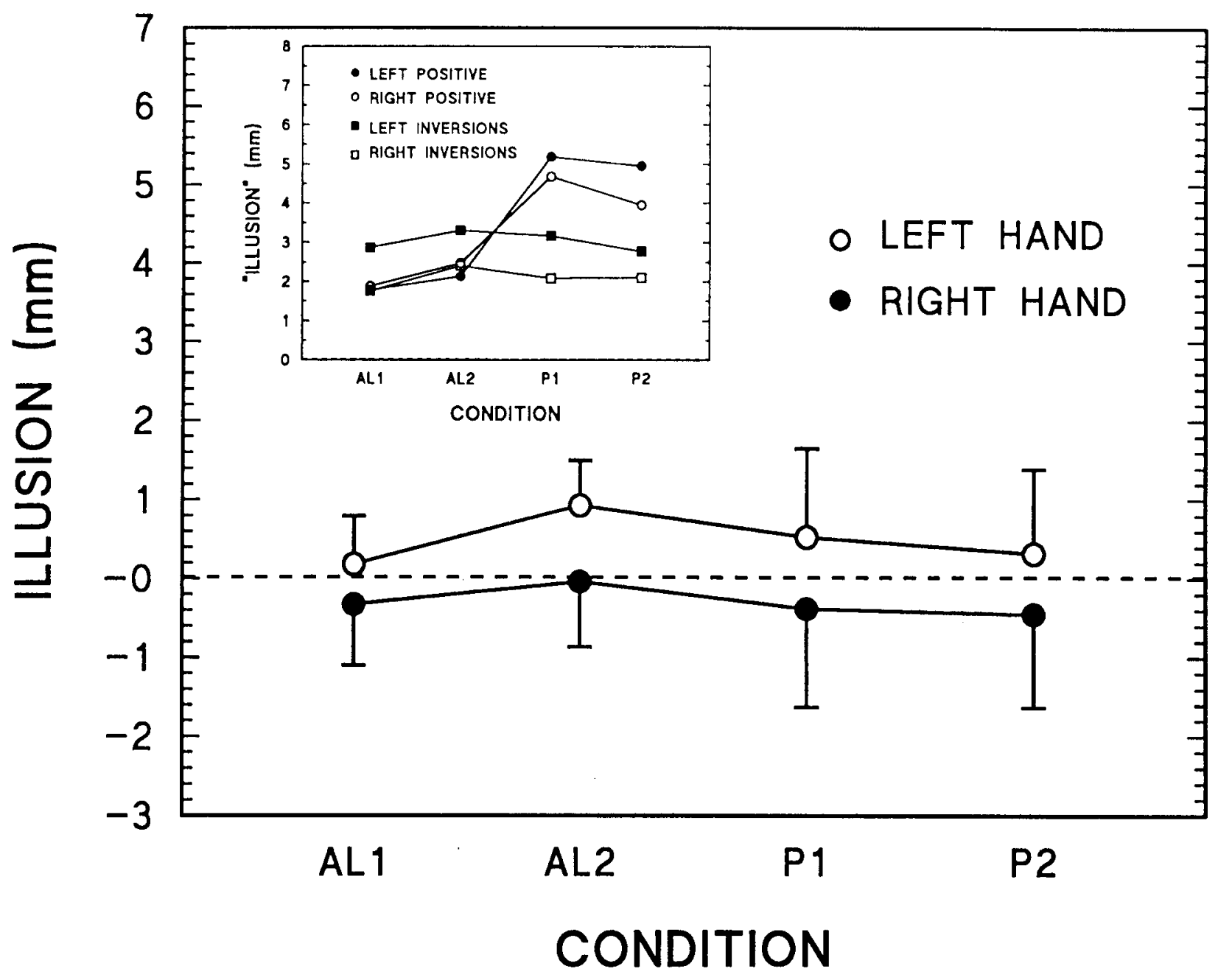

Figure 3. Means and standard errors of illusions obtained in Experiment 1. Error bars show \pm 1 standard error. Inset: positive and "inversion" ("illusion") effects plotted separately.

dispensed with the time limit and reversed the direction of exploration. We also decided to attempt to increase precision by using a simple staircase procedure (for which a time limit would at any rate have been meaningless) and by using only the two more natural conditions of stimulus orientation and exploring hand-namely, $\mathrm{P} 1$ and $\mathrm{AL} 1$ with the left hand and P2 and AL2 with the right.

\footnotetext{
Method

Apparatus and Procedure. The apparatus and stimuli were identical to those of Experiment 1. Step sizes for the staircases were selected to be approximately one standard deviation to begin with, using the data of Experiment 1, and they were halved after three reversals. The point of subjective alignment was estimated from the values of the next six reversals. Step sizes were 4 and $2 \mathrm{~mm}$ for the AL displays and 6 and $3 \mathrm{~mm}$ for the $P$ displays. In each case, the staircase starting position was randomly 2.5 large step sizes above or below true alignment.

The four conditions, AL1-left, P1-left, AL2-right, and P2-right, were presented in random order with a short rest after each condition (approximately every $12 \mathrm{~min}$ ).
}

Subjects. Eleven subjects, all right-handed, were drawn from the same population as in Experiment 1.

\section{Results}

Means and standard errors are shown by the filled circles in the main body of Figure 5. None of these means was significantly different from zero, using each mean's own standard error and one-tailed $t$ tests, with $p(12)>.05$ in each case.

\section{EXPERIMENT 3}

Observation of the subjects' behavior in Experiments 1 and 2 , as well as informal discussion, suggested that the arm holders used tended to hinder the subjects' attempts to align the transversals. Lucca et al. (1986) reported that their subjects' arms were held still by "elastic bands." They did not report how still the forearms were, however, and it may be that our restraining device 
A
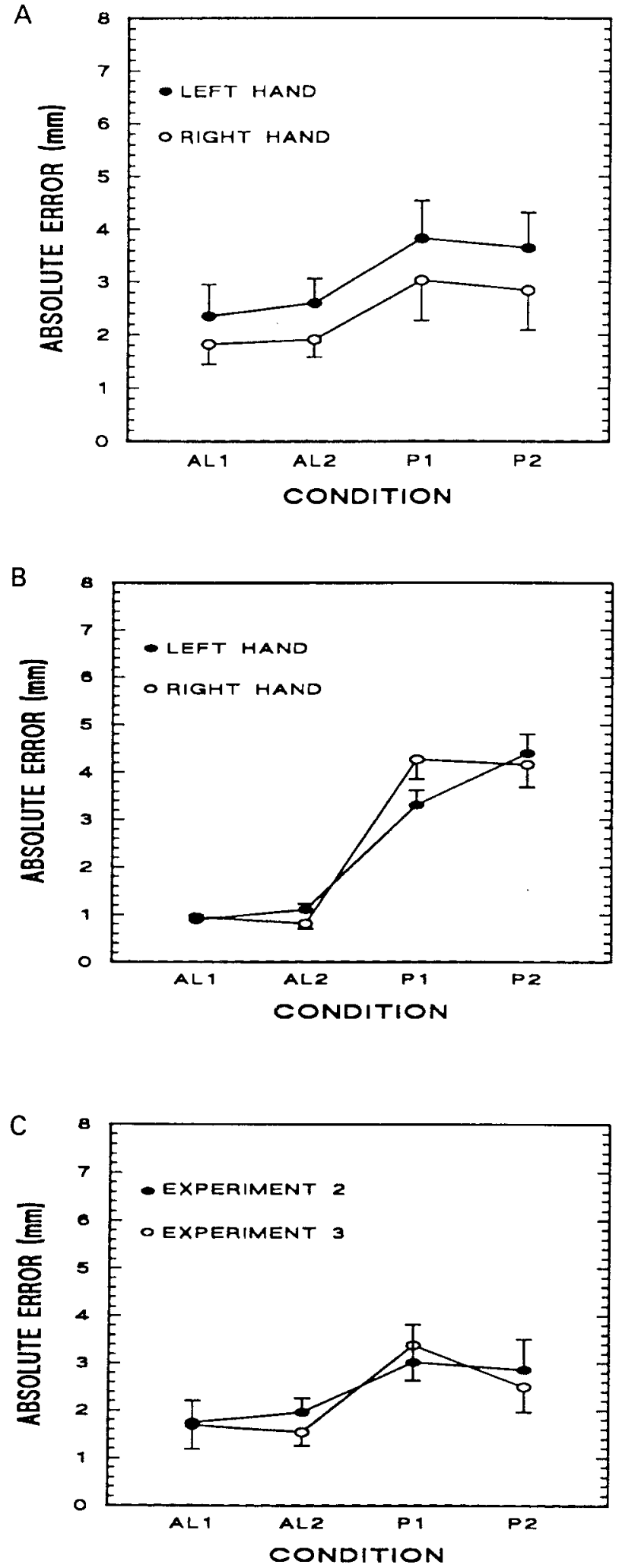

Figure 4. (A) Absolute errors in Experiment 1. (B) Absolute errors reported by Lucca, Dellantonio, and Riggio (1986). (C) Absolute errors obtained in Experiments 2 and 3. was simply too prohibitive. As far as we were concerned, our sole reason for using the restraint had been to replicate the Lucca et al. conditions as closely as possible in Experiment 1.

\section{Method}

Experiment 3 was identical to Experiment 2, except that no restraining device was used. There were 13 subjects, drawn from the same population as before.

\section{Results}

Mean alignment errors are shown by the open circles in Figure 5. One-tailed $t$ tests for single means showed that although the AL2 and P2 means were not different from zero $[t(12)=-.59$ and -.39 , respectively, $p>.05]$, the AL1 and P1 means were nonzero [ $t(12)$ $=-2.05$ and $-2.03, p<.05$ ]. However, it can also be noted that whatever the factor is that contributes to this negative or "inversion" effect for the left hand only, it has its effect in both the absence (AL1) and the presence (P1) of the parallels. Thus, on a conventional test-minuspretest definition of the Poggendorff effect, the difference between P1 and AL1-namely, $0.92 \mathrm{~mm}$-showed no significant Poggendorff illusion in a simple subjects $\times$ treatments analysis of variance $[F(1,36)=0.71, p>.05]$.

At this point, it is instructive to compare the results of Experiments 2 and 3. Figure $4 \mathrm{C}$ shows the absolute errors for both experiments. It can be seen that, relative to the errors in Figure 4A, the errors are smaller than those obtained in Experiment 1 and they are more similar to those of Lucca et al. (1986); this is true for both means and for standard errors. We believe that the larger absolute error and associated standard errors in Experiment 1 were due primarily to the 15 -sec time limit imposed on the subjects. It can be noted that most of our undergraduate subjects were women, so that the proportion of women in our samples was always of the order of $80 \%-90 \%$. Lucca et al. used men only. This difference between the experiments is a possible contributing factor to the different results. Whatever the case, it is clear that the difference disappears with the removal of the time limit, the change to a staircase procedure, and the use of the two more natural combinations of stimulus orientation and hand use.

Figure 5 shows that mean alignment errors were generally more positive in Experiment 2 and more negative in Experiment 3 . This suggests that taking separate measures of positive and "inversion" absolute errors might reveal larger positive than "inversion" errors in Experiment 2 (similar to the finding in Experiment 1; see Figure 3, inset) but the reverse-larger "inversion" than positive errors-in Experiment 3. Figure 5 (inset) demonstrates that this was the case.

\section{EXPERIMENT 4}

Informal observations suggested to us that the twofinger exploration of the stimulus displays used by Lucca 


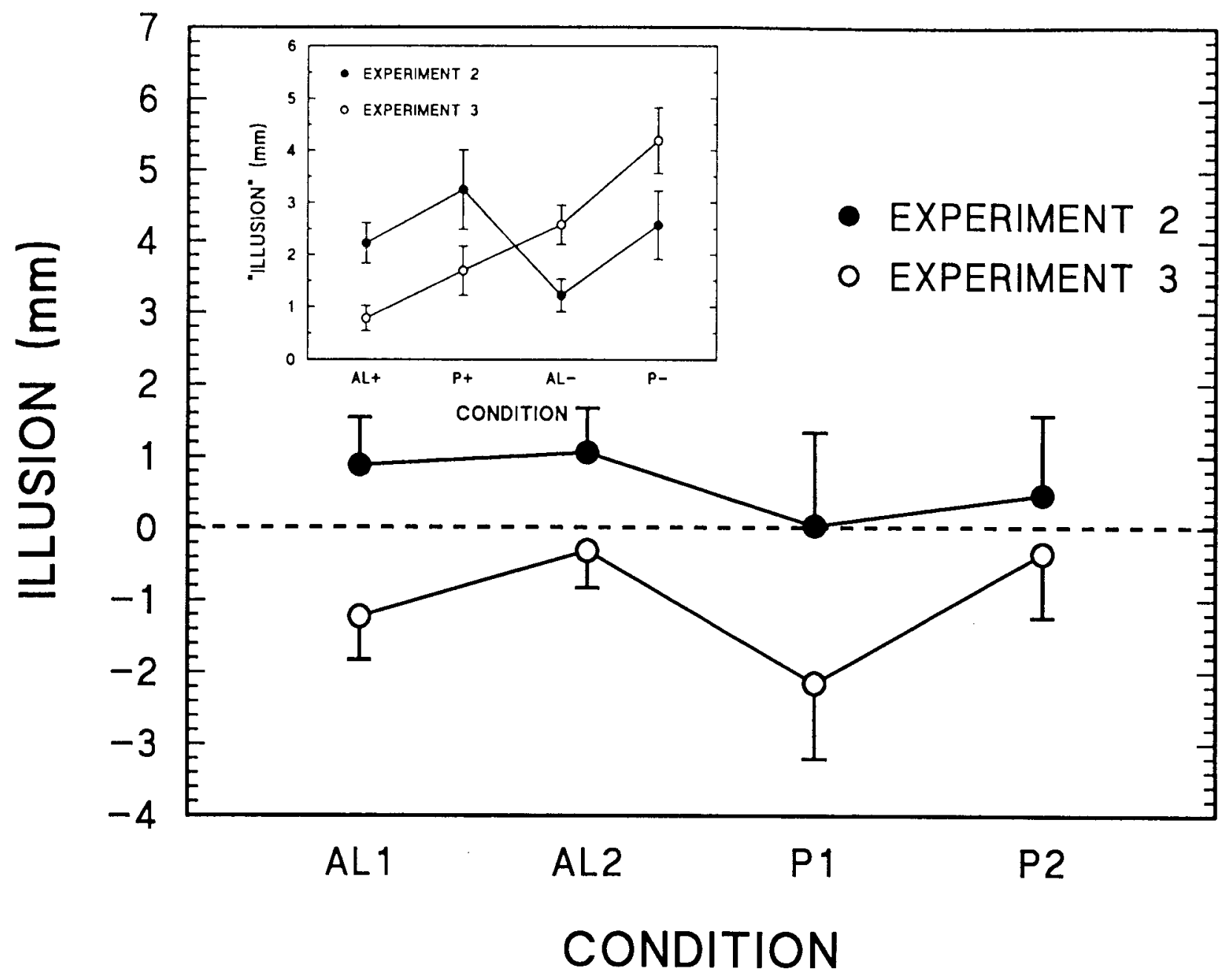

Figure 5. Mean illusions obtained in Experiments 2 and 3. Inset: positive $(+)$ and "inversion" $(-)$ illusions obtained under $A L$ and $P$ conditions in Experiments 2 and $3 . \mathbf{A L}+$ and $P+$ are positive effects; $A L-$ and $P-$ are "inversions."

et al. (1986) was not ideal. Three-finger exploration would provide a more stable probe and also allow simultaneous tactual comparison of the transversal tip on one side of the interparallel gap with the tip on the other side. In Experiment 4, we used just two displays (AL2 and P2) with right-hand exploration by 1,2 , or 3 fingers.

\section{Method}

All aspects of method were as in Experiment 3. The six conditions were completed in a different random order by each subject. There were 14 subjects from the same population as before.

\section{Results}

None of the mean alignment errors in this experiment (Figure 6) was different from zero, with one-tailed $t(14)$ values having a $p>.05$ in every case. However, there was a clear tendency for the standard errors to decrease as a function of the number of fingers used. This is clear from Figure 6. The variances associated with the $\mathrm{AL}$ means, from $A L-1 F$ to $A L-3 F$, were 6.1, 3.6, and 1.7; and those for the corresponding P means were 18.7, 10.6, and 6.8. Since Lucca et al. (1986) offered no particular justification for two-finger exploration, we chose threefinger exploration in the final experiment.

\section{EXPERIMENT 5}

In all the experiments, we observed a tendency for the subjects' fingers to deflect in direction as the fingers hit the first parallel. For example, if the subject was using the right hand, the raised parallel tended to cause the kind of deflection shown in Figure 7. If subjects were unaware of this deflection, their fingers would arrive at the opposite parallel to find the second transverse segment apparently too low, exactly the opposite of the visual Poggendorff effect and the possible basis for the "inversion" effects observed by Lucca et al. (1986) and by us. Our suspicion that our restraining device was too restrictive in the early experiments would be consistent with this idea; 


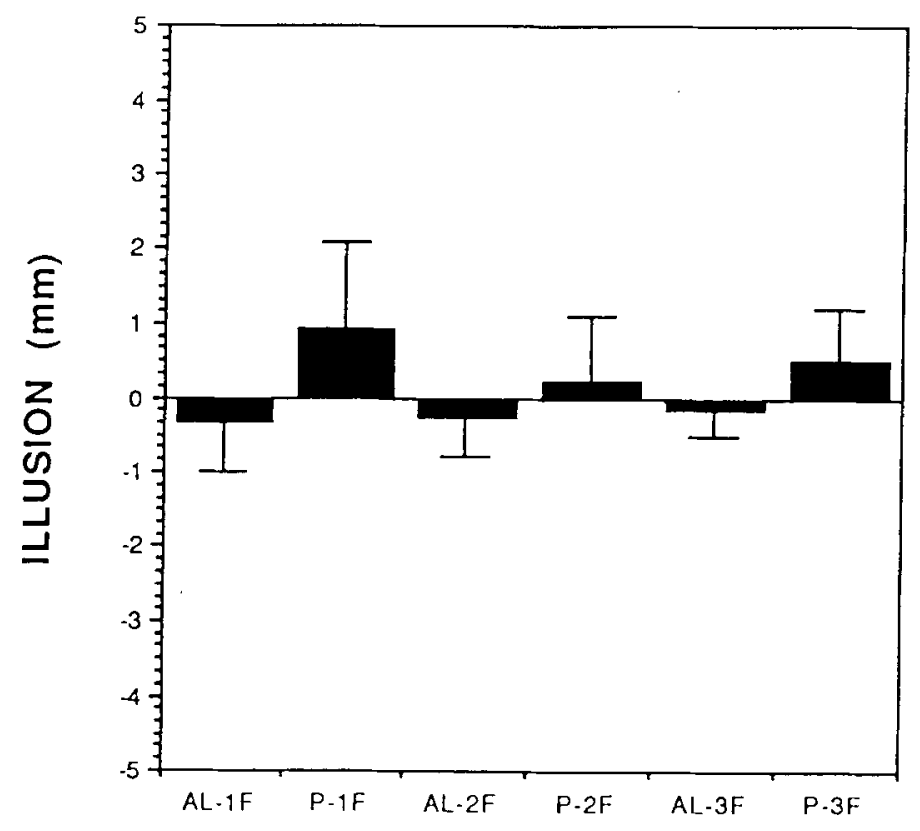

\section{CONDITION}

Figure 6. Mean illusions obtained in Experiment 4.

subjects under restraint would be less able to make this kind of deflection. Consequently, positive errors would be greater than "inversions" under restraint, but the opposite would be the case without restraint. That is exactly what Figures 3 (inset) and 5 (inset) demonstrate. Consistent with this suggestion is the finding of Berla and Butterfield (Berla, 1982; Berla \& Butterfield, 1977) that blind children erroneously follow lines that intersect a haptic test line when the test line's width is narrow but not when it is broad, because they can then trace the test line on the nonintersected side alone, thus avoiding the intersecting line completely.

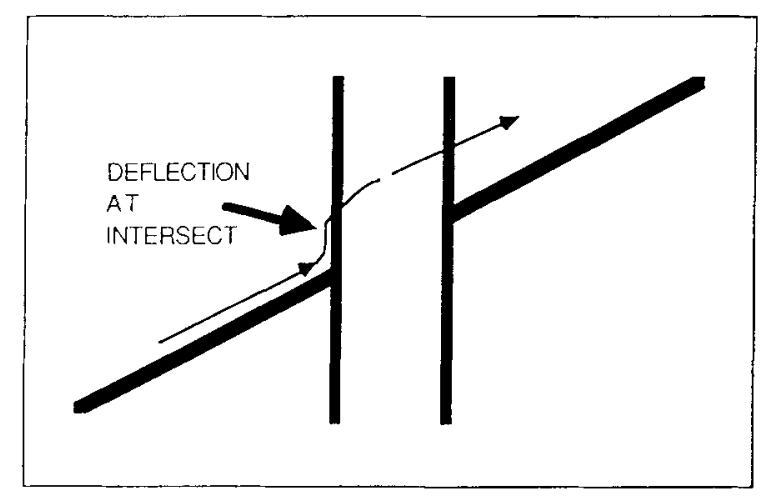

Figure 7, Finger-deflection pattern caused when subject's tracking reaches raised vertical contour.
To test this hypothesis directly, in Experiment 5 we used AL2 and P2 figures only, with one pair of figures exactly the same as those used before. The other pair of figures had grooved or indented rather than raised tracks. The prediction was that "inversions" would be reduced markedly in the absence of raised tracks, because deflections would be less likely to occur.

\section{Method}

Apparatus. The grooved figures were identical in all respects to the raised track figures, except that the grooves were $3 \mathrm{~mm}$ thick. This width was chosen because it was wide enough to prevent deflection along the parallels yet narrow enough to ensure that subjects did not have to lift the fingers out of the groove to transverse the parallel.

Procedure. The four conditions (AL, $P \times$ raised/grooved) were presented in random order. In other respects, the procedure was as in the last experiment, except that subjects always used the three middle fingers for tracking.

Subjects. The 10 subjects were drawn from the usual undergraduate pool.

\section{Results}

The filled circles in Figure 8 (inset) show the mean errors in the four conditions, and the open circles show the mean "inversions." None of the mean errors reached significance by a one-tailed $t(9)$ test, with $p>.05$ in every case. The "inversion" scores were naturally all significant, because they are a subset of all the scores chosen all to be negative. However, it is clear from Figure 8 that the use of grooved stimuli did reduce "inversions." Fi- 


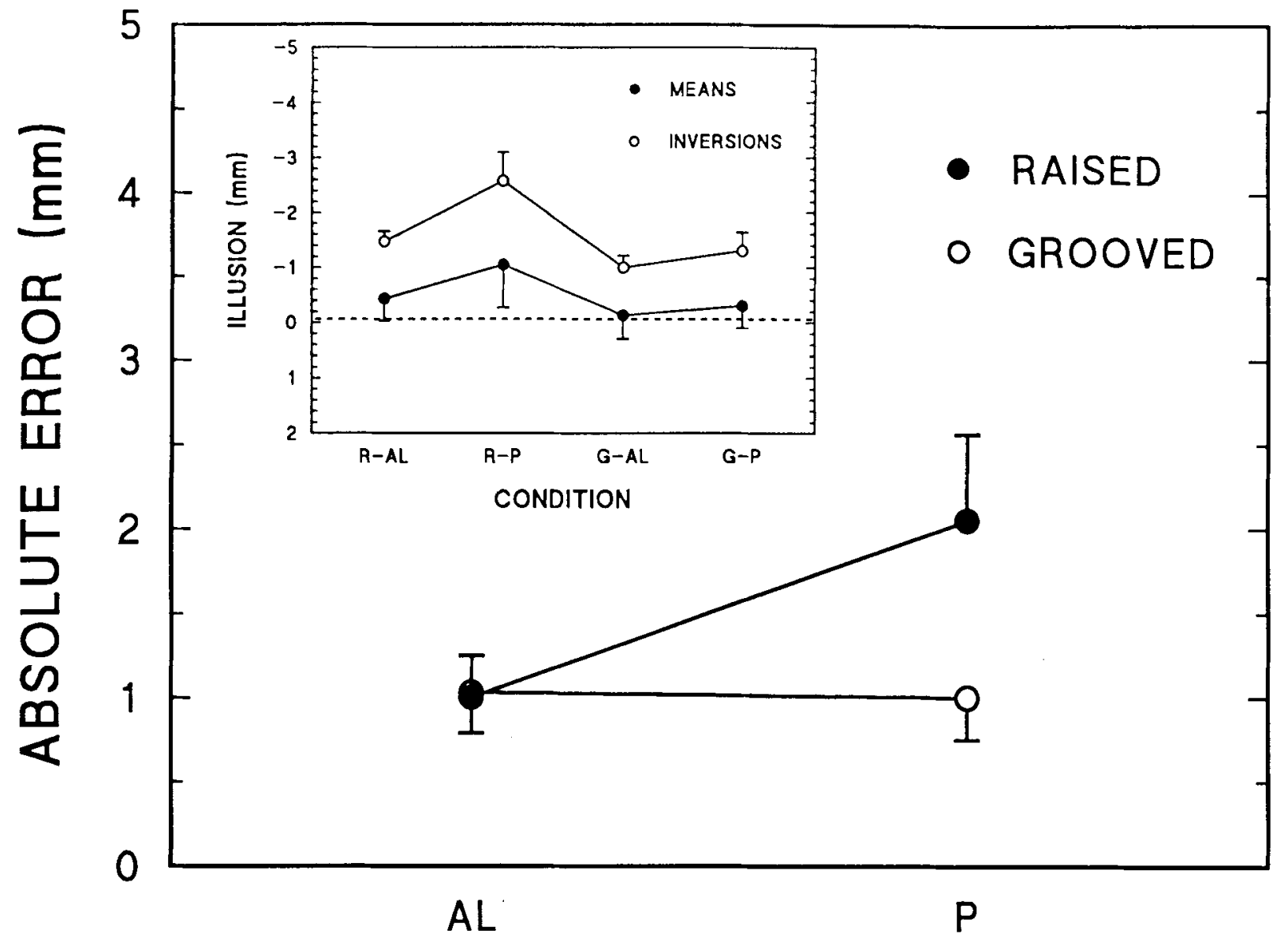

\section{CONDITION}

Figure 8. Absolute errors obtained in Experiment 5. Inset: mean errors and "inversions" obtained in Experiment 5. On the abscissa, $\mathbf{R}=$ raised; $\mathbf{G}=$ grooved.

nally, the main body of Figure 8 shows the absolute errors obtained in the four conditions. It can be seen that the $\mathrm{AL}$ means were very similar in magnitude to those reported by Lucca et al. (1986): see our Figure 4B. In addition, the mean absolute error was also of the order of $1 \mathrm{~mm}$ in the grooved $\mathbf{P}$ condition. Only in the raised $P$ condition, where substantial "inversions" occurred (Figure 8, inset) was the absolute error substantially larger. One-tailed $t$ tests showed that, although the errors in the raised and grooved AL conditions were not different $[t(9)=0.107$ and $p>.05]$, errors in the two $\mathrm{P}$ conditions were different $[t(9)=1.94, p<.05]$. Thus, the larger absolute errors obtained in the raised $\mathrm{P}$ as against the raised AL conditions are essentially due to "inversions" produced, we assert, by physical finger deflections that are unrelated to the Poggendorff effect.

When account is taken of this artifact by using grooved tracks, the mean size of the tactual Poggendorff illusion, defined as test (P) minus pretest ( $\mathrm{AL})$, is $-0.17 \mathrm{~mm}$, a result that must cast doubt on the existence of a tactual analogue to the visual effect and on the claim that the tactual effect exhibits significant "inversion."

\section{GENERAL DISCUSSION}

Lucca et al. (1986) claimed to have demonstrated a tactual analogue of the visual Poggendorff illusion, and they claimed that the tactual effect frequently exhibited effects directionally opposite to the visual effect, which they called "inversions." We have argued that the first conclusion is based on unsound analysis procedures and that the second is based on an artifact of using raised tactual stimuli. The latter artifact produces mistracking errors as the fingers meet the raised parallel, which results in the fingers' reaching the distal transversal at a point that is too high, thus causing "inversions" due entirely to the 
physical characteristics of the display. When grooved stimuli were used (Experiment 5), significant "inversions" did not occur. If "inversions" were a fundamental characteristic of the tactual Poggendorff illusion, then the nature of the stimulus, raised or grooved, should not have affected their occurrence.

Apart from this issue, Lucca et al. (1986) also used absolute rather than mean errors in their analysis. Absolute errors reflect more the dispersion of scores than their central tendency. Over five experiments, despite the occurrence of artifactual "inversions" in some of them, we have obtained 12 different mean Poggendorff illusions, using two- or three-finger tracking. The mean of these 12 mean illusions is $-0.21 \mathrm{~mm}$, and with a standard error of 0.15 , this is not significantly different from zero $[t(11)=1.39, p>.05]$. We conclude that, at least under the conditions of our experiments, no tactual analogue to the Poggendorff illusion is demonstrable.

One possible reason for the nonoccurrence of a tactual illusion could be that tactual exploration in these experiments was necessarily sequential. It might be argued that simultaneous comparison of the left and right obliques is a necessary condition of the illusion. However, that this is not the case was demonstrated by Wenderoth and Johnson (1983), who showed that alternation of the obliques in the visual domain produces a significant illusion without parallels and an enhanced effect with parallels, just as in the case of the more usual simultaneous display.

One factor that might profitably be explored in future research is the effect of visualization on the tactual Poggendorff effect. Intuitively, it seems very unlikely that our subjects completed the task by visualizing a complete Poggendorff display, partly because they were never shown one and partly because of the complexity of the figure. To visualize a Poggendorff figure is not as simple as it is to visualize a single line or rod, as was done by Appelle and Gravetter's (1985) subjects in their estimations of the haptic oblique. However, it remains to be seen whether a tactual Poggendorff analogue might emerge when subjects are shown the complete visual figure at the outset and then are instructed either to use visual imagery during the tactual task or not to do so. Such research might be important for theory. For example, Gillam (1971) has suggested a depth-processing account of the Poggendorff illusion. It may be that there are no tactual analogues of visual depth cues in tactile figures such as those we have used. If an illusion were to emerge with visual imaging, this would add credence to theories of the effect couched in terms of visual mechanisms, including depth processing.

Finally, not only Appelle and Gravetter (1985) but also Heller (1989) draw attention to the fact that, given the normal superiority and heavy use of vision for pattern perception, most normal subjects have limited experience with tactile perception, and they are unable to use it ef- fectively. It would therefore also be of interest to train normal observers in tactile perception of patterns and then to examine their performance on the tactile Poggendorff task. It is possible that training might enhance the use of more global tactile strategies, which, when combined with visual imagery, could produce a tactile analogue of the Poggendorff effect. Relevant to this suggestion is an experiment by Frisby and Davies (1971), who examined the correlation between individuals' settings of visual and haptic Müller-Lyer illusions and concluded that "practice or visual experience (or both) of the haptic device is necessary for the predicted relationships to emerge" (p. 465).

\section{REFERENCES}

APPelle, S., \& GravetTer, F. (1985). Effect of modality-specific experience on visual and haptic judgment of orientation. Perception, 14, 763-773.

BEAN, C. H. (1938). The blind have optical illusions. Journal of Experimental Psychology, 22, 283-289.

BERLA, E. P. (1982). Haptic perception of tangible graphic displays. In W. Schiff \& E. Foulke (Eds.), Tactual perception: A source book (pp. 364-386). New York: Cambridge University Press.

BERLA, E., \& BUTTERFIELD, L. (1977). Tactile political maps: Two experimental designs. Journal of Visual Impairment \& Blindness, 71, 262-264.

Frisby, J. P., \& Davies, I. R. L. (1971). Is the haptic Müller-Lyer a visual phenomenon? Nature, 231, 463-465.

Gillam, B. (1971). A depth processing theory of the Poggendorff illusion. Perception \& Psychophysics, 10, 211-216.

Hatwell, Y. (1960). A study of geometric tactile illusion among the blind. Année Psychologique, 1, 11-27.

Heller, M. A. (1984). Active and passive touch: The influence of exploration time on form recognition. Journal of General Psychology, 110, 243-249.

Heller, M. A. (1989). Picture and pattern perception in the sighted and the blind: The advantage of the late blind. Perception, 18, 379-389.

Lucca, A., Dellantonio, A., * Riggio, L. (1986). Some observations on the Poggendorff and Müller-Lyer tactual illusions. Perception \& Psychophysics, 39, 374-380.

Over, R. (1968). Explanations of geometrical illusions. Psychological Bulletin, 70, 545-562.

Pasnak, R., \& Ahr, P. (1970). Tactual Poggendorff illusion in blind and blindfolded subjects. Perceptual \& Motor Skills, 31, 151-154.

Patterson, J., \& Deffenbacher, K. (1972). Haptic perception of the Müller-Lyer illusion by the blind. Perceptual \& Motor Skills, 35, 819-824.

RÈvÈsz, G. (1934). System der optischen und haptischen Raumtauschungen. Zeitschrift fur Psychologie, 131, 292-375.

TSAI, L. S. (1967). Müller-Lyer illusion by the blind. Perceptual \& Motor Skills, 25, 641-644.

WeNDEROTH, P. (1974). The distinction between the rod-and-frame illusion and the rod-and-frame test. Perception, 3, 205-212.

Wenderoth, P., \& Johnson, M. (1983). Relationships between the kinetic, alternating-line, and Poggendorff illusions: The effects of interstimulus interval, inducing parallels, and fixation. Perception \& Psychophysics, 34, 273-279.

WITKIN, H. A., \& Asch, S. E. (1948). Studies in space orientation: IV. Further experiments on perception of the upright with displaced visual fields. Journal of Experimental Psychology, 38, 762-782.

(Manuscript received November 20, 1989; revision accepted for publication March 29, 1990.) 